\title{
Solar Spectral Proxy Irradiance from GOES (SSPRING): a model for solar EUV irradiance
}

\author{
Katherine Suess ${ }^{1}$, Martin Snow ${ }^{1, *}$, Rodney Viereck ${ }^{2}$, and Janet Machol ${ }^{3,4}$ \\ 1 Laboratory for Atmospheric and Space Physics (LASP), University of Colorado, Boulder, CO 80309, USA \\ *Corresponding author: Marty.Snow@lasp.colorado.edu \\ 2 Space Weather Prediction Center (SWPC), National Oceanic and Atmospheric Administration, Boulder, CO 80305, USA \\ 3 Cooperative Institute for Research in Environmental Sciences (CIRES), University of Colorado, Boulder, CO 80309, USA \\ 4 National Centers for Environmental Information, National Oceanic and Atmospheric Administration, Boulder, CO 80305 , \\ USA
}

Received 28 April 2015 / Accepted 3 January 2016

\begin{abstract}
Several currently operating instruments are able to measure the full EUV spectrum at sufficient wavelength resolution for use in upper-atmosphere modeling, the effects of space weather, and modeling satellite drag. However, no missions are planned at present to succeed the Thermosphere Ionosphere Mesosphere Energetics and Dynamics (TIMED) and Solar Dynamics Observatory (SDO) missions, which currently provide these data sources. To develop a suitable replacement for these measurements, we use two broadband EUV channels on the NOAA GOES satellites, the magnesium core-to-wing ratio (Mg II index) from the SOlar Radiation and Climate Experiment (SORCE) as well as EUV and Mg II time averages to model the EUV spectrum from 0.1 to $105 \mathrm{~nm}$ at $5-\mathrm{nm}$ spectral resolution and daily time resolution. A Levenberg-Marquardt least squares fitting algorithm is used to determine a coefficient matrix that best reproduces a reference data set when multiplied by input data. The coefficient matrix is then applied to model data outside of the fitting interval. Three different fitting intervals are tested, with a variable fitting interval utilizing all days of data before the prediction date producing the best results. The correlation between the model results and the observed spectrum is found to be above $95 \%$ for the $0.1-50 \mathrm{~nm}$ range, and between $74 \%$ and $95 \%$ for the $50-105 \mathrm{~nm}$ range. We also find a favorable comparison between our results and the Flare Irradiance Spectral Model (FISM). These results provide a promising potential source for an empirical EUV spectral model after direct EUV measurements are no longer available, and utilize a similar EUV modeling technique as the upcoming GOES-R satellites.
\end{abstract}

Key words. Solar irradiance - EUV flux - Space weather

\section{Introduction}

Fluctuations in solar irradiance drive variations in many upper atmospheric processes including satellite drag, total electron content, ground-space communications, and GPS precision. These variations are highly wavelength dependent, and below 200-nm irradiances can change by more than an order of magnitude over the course of a solar cycle (Woods et al. 2012). The solar output at these shorter wavelengths can also vary greatly on much smaller timescales than that of the 11-year solar cycle. Active regions rotate on and off the solar disk with the Sun's 27-day rotation, and flares happen over the course of minutes to hours. Due to the large degree of variability in the shorter wavelengths, it is highly desirable to have a reliable data source in this wavelength range to facilitate accurate predictions of the effects of solar EUV irradiance changes on Earth.

Several satellites currently measure irradiance at the far and extreme ultraviolet (FUV and EUV) wavelengths, which range from approximately 0.1 to $120 \mathrm{~nm}$. The Thermosphere Ionosphere Mesosphere Energetics and Dynamics Solar EUV Experiment (TIMED SEE) measures the solar spectrum from 0.5 to $190 \mathrm{~nm}$ at $0.5 \mathrm{~nm}$ resolution (Woods et al. 2005). The Solar Dynamics Observatory EUV Variability Experiment (SDO EVE; Woods et al. 2012) also provides coverage of EUV spectral irradiance, at even finer wavelength resolution. However, both TIMED (launched in 2001) and SDO (launched in 2010) are research missions, not operational ones; there will likely be no replacement instruments after these missions end, unlike the case for operational satellites such as the NOAA Geostationary Operational Environmental Satellites (GOES). It is therefore important to develop accurate models of the EUV spectrum that can be relied on when direct data sources are no longer available. In this paper, we introduce the Solar Spectral Proxy IrradiaNce from GOES (SSPRING), an empirical model for EUV irradiance that uses data from NOAA GOES and the NASA SOlar Radiation and Climate Experiment (SORCE; Rottman 2005).

SSPRING models EUV irradiance in 5-nm bins stretching from 0.1 to $105 \mathrm{~nm}$. The model is built from three wavelength measured bands as well as their time averages: two GOES EUV bands, the magnesium core-to-wing ratio (Mg II index) from SORCE, and their 40-day trailing averages. We use a Levenberg-Marquardt least squares fitting algorithm (mpfit for IDL, Markwardt 2009) to find the linear combination of these six input data sources that best matches reference data sources from TIMED SEE and SDO EVE. We test three fitting intervals and compare the results with the FISM for EUV irradiance. 


\subsection{Existing EUV proxies and models}

A variety of proxies and models for EUV irradiance already exist. The sunspot number has been measured for hundreds of years and can provide an estimate for overall solar activity during solar maximum. During solar minimum, the inability of sunspot number to go below zero leads to decreased accuracy of predictions for many EUV wavelengths.

The F10.7 index, the solar radio flux at $10.7 \mathrm{~cm}$, is also a common solar proxy (Tapping 2013). F10.7 can penetrate the atmosphere and be measured by ground-based observatories (unlike EUV). As a result, F10.7 has been measured since before the space era and a continuous data set is available from 1947 to the present. Other radio wavelengths, such as 3.2, 8, 10,15 , and $30 \mathrm{~cm}$, can also serve as proxies for solar activity (Dudok de Wit et al. 2014). Before EUV data was available from space-based observatories, F10.7 was one of the most common proxies for the EUV. However, much like sunspot number, it is not directly correlated to EUV irradiance. Instead, it is produced through up to three emission mechanisms occurring in the chromosphere and corona, including thermal Bremsstrahlung, gyromagnetic resonance, and possible nonthermal emissions (Tapping 2013). Like the sunspot number, F10.7 appears to have a lower limit during solar minimum (Viereck et al. 2001).

The magnesium II core-to-wing ratio (Mg II index), another solar EUV proxy, is the ratio of the $h$ and $k$ lines of the magnesium flux near $280 \mathrm{~nm}$ to the background solar continuum around these lines (Heath \& Schlesinger 1986). This proxy does not exhibit the decreased accuracy during solar minimum seen in the F10.7 and sunspot number proxies. Since the $\mathrm{Mg}$ II index is a ratio, it is also less sensitive to artifacts and instrument degradation than a non-ratio measurement.

Existing empirical and semi-empirical models include the EUV81 model (Hinteregger \& Fukui 1981), the EUVAC model (Richards et al. 1994, 2006), the SOLAR2000 model (Tobiska et al. 2000; Tobiska 2004), the NRLEUV model (Warren et al. 2001; Lean et al. 2003; Warren 2005), the FISM (Chamberlin et al. 2007), and the empirical model presented in Fontenla et al (2014). Chamberlin et al. (2007) compare the first four listed models with TIMED SEE data and determine that the models differ from SEE data by $20-47 \%$ in the $35-40 \mathrm{~nm}$ band during a rotation in January 2004. FISM uses one proxy per 1-nm wavelength bin to model rotational and baseline irradiance components, and achieves higher correlation with SEE data than the EUVAC, EUV81, and SOLAR2002 models (Chamberlin et al. 2007). Two major differences differentiate SSPRING from FISM: SSPRING uses multiple proxies per wavelength bin and does not individually model the baseline and rotational irradiance components. Cessateur et al. (2011) construct a full EUV spectrum from observations in a few passbands similar to the methods used in this paper.

\section{Input data sets}

The input data sets which drive SSPRING approximately emulate the measurements that will be made on the upcoming GOES-R satellites and include operational data sources that span the EUV range to be modeled. The input data sets (Table 1) provide representative information about the corona, chromosphere, and transition region. This captures a large amount of the variability in the processes producing EUV radiation, allowing for more accurate EUV modeling.
Table 1. Data sources used, the measured feature, and the wavelength range of the measurement. Four GOES data products and one SORCE SOLSTICE data product are utilized. In addition, a 40-day trailing average is included for all inputs.

\begin{tabular}{lcc}
\hline \hline Data Source & $\begin{array}{c}\text { Measured } \\
\text { feature }\end{array}$ & $\begin{array}{c}\text { Wavelength } \\
\text { range }\end{array}$ \\
\hline GOES-15 EUVS-B (v2) & He II & $26-34 \mathrm{~nm}$ \\
GOES-15 EUVS-E (v2) & Ly- $\alpha$ & $118-122 \mathrm{~nm}$ \\
SOLSTICE Mg II (v13) & Mg II & $280 \mathrm{~nm}$ \\
Trailing Avg EUVS-B & Time Avg He II & $26-34 \mathrm{~nm}$ \\
Trailing Avg EUVS-E & Time Avg Ly- $\alpha$ & $118-122 \mathrm{~nm}$ \\
Trailing Avg Mg II & Time Avg Mg II & $280 \mathrm{~nm}$ \\
\hline
\end{tabular}

Operational broadband measurements of the EUV spectrum are available from the NOAA GOES satellites (Viereck et al. 2007). We use measurements from GOES-15; this satellite covers the entire modeled time period, so there is no need to merge with GOES-13 or -14 data. GOES EUVS-B and -E bands capture the spectrum around the He II (EUVS-B) and Lyman-alpha (Ly- $\alpha$ ) (EUVS-E) lines, two of the strongest solar emissions. The GOES channel E data is converted to a 1-nm band around the Lyman- $\alpha$ line at $121.6 \mathrm{~nm}$. Based on the LASP WHI quiet Sun reference spectrum (Woods et al. 2009), about $88 \%$ of the full channel irradiance is contained in the 1-nm band. To correct for degradation in the E channel, the GOES data is scaled to SORCE SOLSTICE (McClintock et al. 2005) Lyman- $\alpha$ measurements with a double exponential function. This correction indicates that for GOES-15, channel E degraded by $11 \%$ after 1 year and $32 \%$ after 5 years, while GOES-13 and -14 had slower degradation rates. The new generation of satellites will no longer measure broadband channels as the current GOES model does, but the central emission features should be similar enough to allow SSPRING to adapt smoothly to the new data source. GOES-R will also measure the $\mathrm{Mg}$ II index, allowing us to continue using this proxy after the end of the SORCE mission.

We also use the Mg II index from the SORCE SOLSTICE instrument (Snow et al. 2005). SOLSTICE measures the spectrum around $280 \mathrm{~nm}$ with $0.1 \mathrm{~nm}$ resolution, and this highresolution index has a smaller percent error than many other available EUV proxy indices (Snow et al. 2005). The Mg II index has been shown to be a better proxy for solar EUV irradiance than the F10.7 index (Viereck et al. 2001), in part due to the magnesium proxy's continued accuracy during solar minimum.

In addition to the daily average value for EUVS-B, EUVS-E, and $\mathrm{Mg}$ II, we also use time averages. The time average models the long-term solar variation and minimizes the impact of solar rotation. Many models use an 81-day centered average as a component of the F10.7 index to more accurately model solar cycle variability (Hinteregger \& Fukui 1981; Viereck et al. 2001); however, the use of this centered average makes real-time use of the proxy impractical. If SSPRING is to be used in near real time, we must use a trailing average. Figure 7 of Chamberlin et al. (2007) shows that a trailing average of 40 days produces a 0.93 correlation between the $\mathrm{Mg}$ II proxy and the modeled 1-nm irradiance. Doubling the smoothing interval to 80 days only increases the correlation to 0.94 , so a longer smoothing interval yields rapidly diminishing returns. Therefore we have chosen a 40-day trailing average for this version of our model.

There are several data gaps within the modeled time period, none longer than 2 weeks. During data gaps, a linear 
interpolation of the data was performed. The days that used interpolated reference data were used only to generate the time averaged inputs and not included in the analysis of how well the model performed against the reference data.

\section{Reference data sets}

TIMED SEE and SDO EVE data sets are used both to create the SSPRING coefficients and to test the model's accuracy. SDO EVE, the Solar Dynamics Observatory's EUV Variability Experiment, was launched in 2010 as a part of NASA's Living with a Star Program. EVE measures EUV irradiance at $0.1 \mathrm{~nm}$ resolution with a 10-second time cadence (Woods et al. 2012), allowing us to capture solar flares. However, more rapid than expected degradation of the MEGS-B instrument, which measures the longer-wavelength portion of the EUV spectrum, led to decreased calibration accuracy of the spectrum greater than $40 \mathrm{~nm}$ (EVE News 19 May 2010). Furthermore, a power anomaly rendered measurements from MEGS-A unavailable after May 26, 2014 (EVE News 28 May 2014).

Due to the degradation of MEGS-B during the test time period, we used data from TIMED SEE, the NASA Thermosphere Ionosphere Mesosphere Energetics and Dynamics mission's Solar EUV Experiment instrument, for wavelengths past $40 \mathrm{~nm}$. SEE, launched in 2001, observes the Sun for approximately 3 min of every 97 min orbit (Woods et al. 2005). SEE does not observe the Sun continuously enough to provide good data for solar flares, which can occur on timescales much shorter than TIMED's orbital period. Due to this orbital limitation, the Level 3 "daily" SEE data product we use in our calculations has had flares removed by the SEE team (Woods et al. 2005).

While the differences in the cadence and resolution of the SEE and EVE data sets are fairly large, the model construction is not sensitive to the differences. Modeling a daily EUV spectrum as we present here as opposed to a minute-by-minute one makes the time cadence differences between the SDO and TIMED spacecraft much less important, as both instruments have enough sample points per day to produce a reliable daily average. Additionally, both instruments have much finer wavelength resolution than $5 \mathrm{~nm}$, rendering differences in spectral resolution of the two instruments unimportant for SSPRING's construction.

The data sets we used for each part of the spectrum are presented in Table 2. This combination allowed coverage of the EUV spectrum and use of the most accurate available data for the wavelength range.

\section{Model construction}

We created equal-sized bins for the first iteration of this model and chose the median size of the Solomon \& Qian (2005) bands of $5 \mathrm{~nm}$ as the bandwidth; a $5-\mathrm{nm}$ spectral resolution is also the requirement for the new GOES-R EUV products (Eparvier et al. 2009). Using the methods employed for SSPRING, it would be simple to determine the coefficients for other modeled bandpasses. Thus, we could easily provide a version of SSPRING with resolution equal to that proposed in Solomon \& Qian (2005) if necessary.

Because the SDO EVE reference data set begins at $6 \mathrm{~nm}$, the first band stretches from 0.1 to $6 \mathrm{~nm}$ and the second from 6 to $10 \mathrm{~nm}$. The remaining 19 bands, starting at $10 \mathrm{~nm}$, have a
Table 2. Reference data used for each wavelength range. The TIMED SEE "Integrated Spectrum" refers to the calibration of Level 3 SEE data short of $27 \mathrm{~nm}$ (Woods et al. 2005). In this range, a solar model is scaled to match broadband data from the instrument, and finer-resolution data is extracted from the model.

\begin{tabular}{ll}
\hline \hline Wavelength range & \multicolumn{1}{c}{ Reference spectrum } \\
\hline $01-6 \mathrm{~nm}$ & TIMED SEE Integrated Spectrum (v11) \\
$6-40 \mathrm{~nm}$ & SDO EVE (v3) \\
$40-105 \mathrm{~nm}$ & TIMED EE (v11) \\
\hline
\end{tabular}

5-nm width. This produces a total of 215 -nm bands from 0.1 to $105 \mathrm{~nm}$. These are similar to the bandpasses that will be produced by the next-generation GOES-R satellites (Eparvier et al. 2009).

The input data set, discussed in Section 2, represents solar flux in Ly- $\alpha$, He II, and the Mg II index- or the solar transition region, chromosphere, and corona. One can think of these different input time series as semi-orthogonal functions, where an active region tends to affect each input in a similar fashion but to a different degree. If the activity levels of the processes producing solar EUV are correctly combined, an accurate proxy for the observed irradiance can be attained. Based on this idea, we model EUV irradiance as a linear combination of each input according to the equation:

$$
\begin{aligned}
\text { EUV irradiance }= & w_{1}+w_{2} \times \text { EUVS-B }+w_{3} \times \text { EUVS-E } \\
& +w_{4} \times \text { EUVS-B trailing average } \\
& +w_{5} \times \text { EUVS-E trailing average } \\
& +w_{6} \times \text { Mg II Index trailing average }
\end{aligned}
$$

where $w_{1}$ through $w_{6}$ refer to the weights produced by the fitting algorithm and "trailing average" refers to a 40-day trailing average or smoothed version of the time series.

We use a Levenberg-Marquardt damped least squares fitting algorithm (mpfit for IDL, Markwardt 2009) to determine the six weights that best produce the reference spectra from a linear combination of the input data time series. As inputs, the algorithm requires: a functional form for the solution (shown in Eq. (1)), the data that makes up each independent variable in the function, a set of data to compare the function to which it serves as a fitting set for the model (SDO EVE and TIMED SEE), and a set of initial parameters for each independent variable in the function.

The fitting was performed with normalized inputs and reference values due to the wide range of magnitudes of the input data. Normalizing the inputs to the same baseline order of magnitude allowed the fitting program to perform fewer iterations with a small, uniform initial parameter, as opposed to attempting to minimize the number of iterations on nonnormalized inputs by a clever choice of initial parameters. This simple normalization used typical values from the middle of our time period. We similarly normalized the reference data sets by dividing the irradiance time series for each 5-nm bin by the irradiance on a particular day. January 1, 2012 was chosen as the normalization day. This normalization produced time series that are all equal to 1.0 on January 1,2012 and vary based on the percent change from that day. The overall input and reference data are not on the same scale - they simply use the same baseline value to aid the fitting program. It is easy to convert the normalized model results back to physical 
irradiance units by multiplying each band by its normalization factor.

The weighting coefficients for each 5 -nm bin were determined separately. With 21 bands and 6 fitting coefficients per band, the result of the fitting algorithm was a $6 \times 21$ matrix of coefficients that best produced the output fitting data from a linear combination of the input data sets. Applying this matrix to input time series outside the fitting interval produced a modeled EUV spectrum. To compare the modeled spectra with reference data, we have so far tested SSPRING only in time periods where both a reference data set and an input data set from the GOES and SORCE satellites are available. This period spans from April 2010 to July 2013.

\subsection{Fitting intervals}

We tested three different fitting intervals. The first two were fixed, and the third was variable. Fitting interval choice can dramatically affect the accuracy of the modeled results for many reasons, so it is important to test different intervals and determine which produces the best results. The fitting interval can affect model accuracy for several reasons: a larger dynamic range in the reference data of the training period typically improves the accuracy of the fit, and the closer the fitting interval is to the dates of interest the less important any drift in the input data or reference data will be. We began by testing two different types of fixed fitting intervals to assess the effectiveness of our model function and fitting algorithm. For almost all bands, the correlation coefficients between the model results and the reference spectra were above $70 \%$ (discussion in Sect. 5.1). These results showed that our overall method was appropriate to model the EUV data. We then moved on to a slightly more complex variable fitting period method, which further improved the model's accuracy.

For the first fixed fitting interval, SSPRING used reference data from either the 2011 or 2012 calendar year, a period long enough to include information about both 27-day solar rotation and some solar cycle changes. Section 5.1 contains the results of this fitting interval.

Next, moving a fitting interval of fixed length through time determined how the fitting interval choice affected the model results. We created model results for the first 2 weeks of February 2013 for 14 different four-month periods which began 2.5 years before the test days and ended with the inclusion of the test days. The results from this analysis are given in Section 5.2.

With the first fixed fitting interval, we determined that the basic concept of the model produced viable results. With the second fixed fitting interval, we verified our prediction that a fitting interval closer to the test dates would produce more accurate results. With the variable fitting interval, we aimed to make a model that combined the successes of the first two fitting intervals and utilized all available data, an approach that best mimics operational use. In this model, daily expansions of the fitting interval made use of all data that would have been available if SSPRING had been run on that day. So that the initial model results did not suffer from a lack of fitting data, all available data from 2010 was used to predict irradiances in 2010. After that, the fitting interval was expanded each day. To model April 3, 2012, for example, the fitting interval spanned from April 1, 2010 (beginning of data set) to April 2, 2012 (day before prediction date). This variable fitting interval model produces more accurate model results than the fixed fitting interval. Furthermore, GOES produces data at a $30-\mathrm{sec}-$ ond cadence for space weather prediction. This makes it possible to run this model daily and obtain accurate estimates for the daily EUV irradiance. While up-to-date reference data will not be available after the SEE and EVE missions are complete, this method of expanding the fitting interval best mimics using all available fitting data, while not including future data in the fit. Section 5.3 details the results of this fitting interval selection.

\subsection{Model uncertainties}

Several main factors contribute to uncertainties in SSPRING. There will be error in the reconstruction, which we quantify in the Results section using the linear Pearson correlation coefficient. Additionally, there are uncertainties in the training data sets as well as the data sets we compare the model to. The formal uncertainty in TIMED SEE and SDO EVE data is $~ 30 \%$, but this includes calibration uncertainties; the day-to-day repeatability is on the order of a few percent (see readme files for SDO EVE and TIMED SEE). Because we compute normalized results that are then pegged to the real value on a specific day, this day-to-day repeatability is the relevant uncertainty; this uncertainty is much less than the uncertainty in the model. Neither TIMED SEE nor SDO EVE cover a full solar cycle- in particular, neither covers the descending phase of the solar cycle. This will lead to additional uncertainty when modeling the descending phase of the solar cycle, but is difficult to quantify in advance. If SSPRING is used after the release of GOES-R data, there will also be uncertainties that result from the change in the wavelength coverage and sensitivity of the EUVS bands. This uncertainty may be calculated after the release of GOES-R data.

\section{Results}

\subsection{Results for a one-year fixed fitting interval}

To test the effectiveness of the models produced by the 2011 and 2012 year-long fitting intervals, we calculated linear Pearson correlation coefficients. For each 5-nm bin, we found the correlation coefficient over the entire data period between the modeled time series and the observed EVE or SEE time series. Figure 1 shows the correlation as a function of wavelength for both the 2011 and 2012 fitting intervals. It is evident from these plots that the correlation is higher in the shorterwavelength bins than in the longer-wavelength bins. In both,

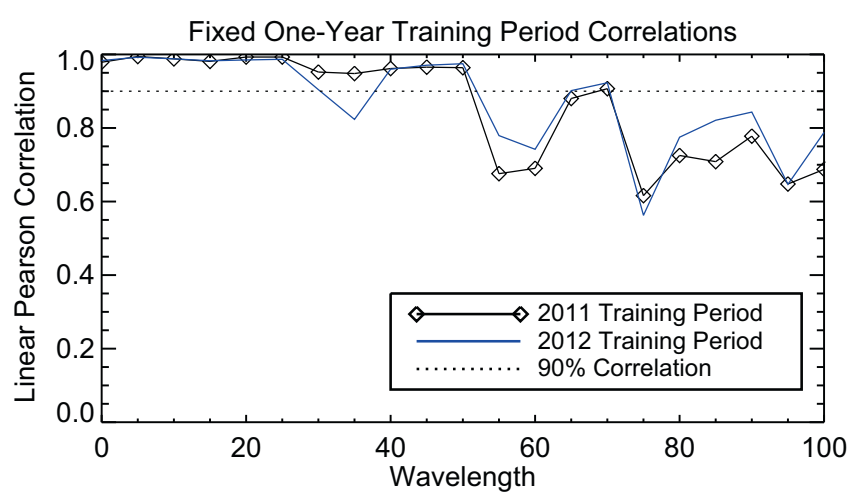

Fig. 1. Data and model correlation for both the 2011 and 2012 fitting intervals. 

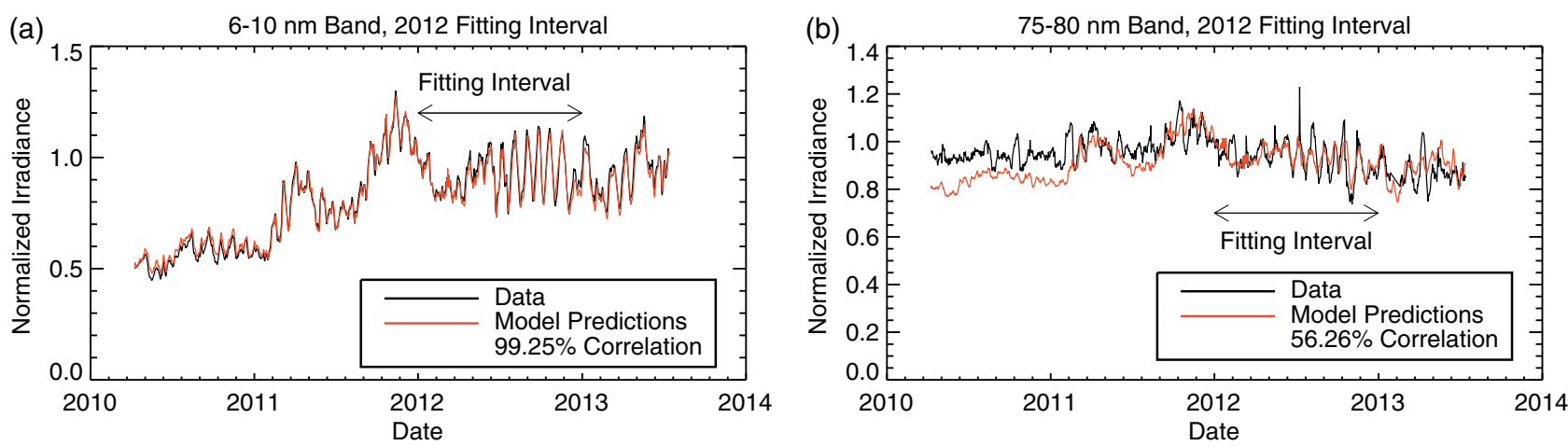

Fig. 2. Example 4-year time series for 2012 training period. Not only does the $6-10 \mathrm{~nm}$ band better capture day-to-day variations, it also more closely follows the overall solar activity level. Note that for the $75-80 \mathrm{~nm}$ band, the farther away from the fitting interval the model results are, the less likely they are to overlay the observed data. Reference data uncertainties are a few percent, on the order of the size of the line used to plot. (a) Strongest correlation for 2012 fitting interval, (b) weakest correlation for 2012 fitting lnterval.
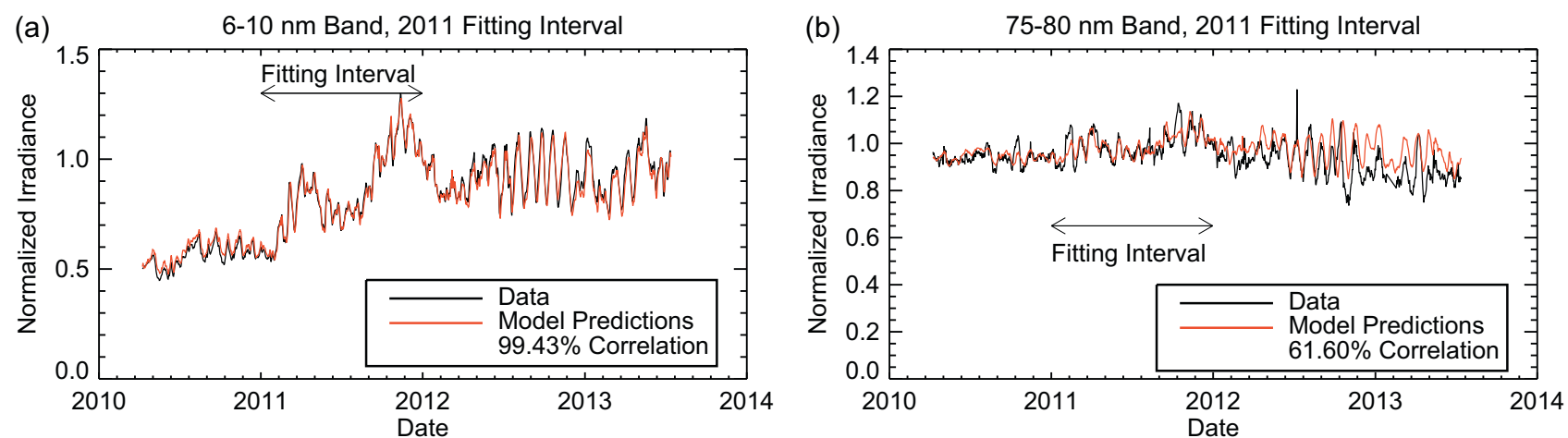

Fig. 3. Example time series for 2011 training period. The highest correlation for this fitting interval looks fairly similar to the 2012 fitting interval, but the $75-80 \mathrm{~nm}$ band has improved by $5 \%$. This is evident as the model results no longer diverge from the observations at the edges of the time sample. Data uncertainties are a few percent, on the order of the size of the line used to plot. (a) Strongest correlation for 2011 training period, (b) weakest correlation for 2011 training period.

the correlation coefficient is fairly constant until approximately $50 \mathrm{~nm}$ but drops off for longer wavelengths. This is likely in part due to our input data. As discussed in Section 2, the input data describes the irradiance in Ly- $\alpha, \mathrm{He}$ II, and Mg II. None of these inputs span the $50-110 \mathrm{~nm}$ range. This likely leads to failure to capture some portion of the variability in this part of the spectrum, causing decreased model accuracy. Furthermore, solar irradiance is both stronger and more variable below $40 \mathrm{~nm}$; thus, both input and reference data have higher signalto-noise values, likely leading to better fits. Because the solar spectrum is less variable above $45 \mathrm{~nm}$ and there is less irradiance at these wavelengths, it is less critical to obtain accurate fits and predictions in this region of the spectrum. The cause of the apparent oscillations in the correlation in Figure 1 is not clear.

In Figures 2 and 3 we plot the time series for the wavelengths with the highest and lowest correlation coefficients in each fitting interval. For both years, the maximum correlation occurs in the $6-10 \mathrm{~nm}$ band, and the minimum occurs in the 75-80 $\mathrm{nm}$ band. However, the correlations for both of these bands are higher for the 2011 fitting interval than for the 2012 fitting interval. Arrows on the plots indicate the fitting interval utilized for each modeled time series. The differences in correlation between the 2 years indicate that the choice of fitting interval does contribute to the overall success of the model; it is therefore important to carefully pick the fitting interval to provide the best results.

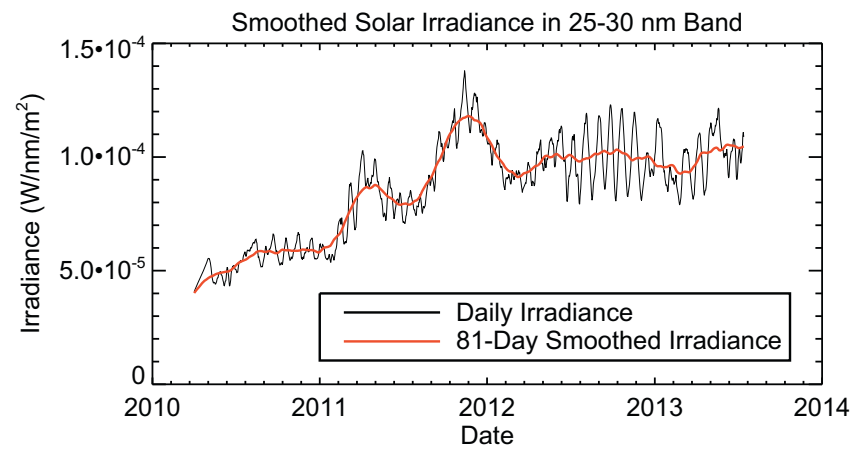

Fig. 4. Daily and smoothed irradiance for $25-30 \mathrm{~nm}$ band. The smooth version allows us to see the larger solar cycle variability in 2011 when compared to 2012, contributing to the 2011 fitting interval's increased accuracy.

It is evident from the red smoothed curve in Figure 4 that the baseline irradiance changes more in 2011 than in 2012. The overall percent change in this band between the first and last day of 2011 is $95 \%$, whereas in 2012 the percent difference is only $12 \%$. This indicates that the 2011 fitting interval contains more information about the variability due to the solar cycle than the 2012 fitting interval and helps explain the 2011 fitting interval model's higher correlation with the observed data. We further examined the effects of the fitting interval choice in Section 5.2. 
(a)

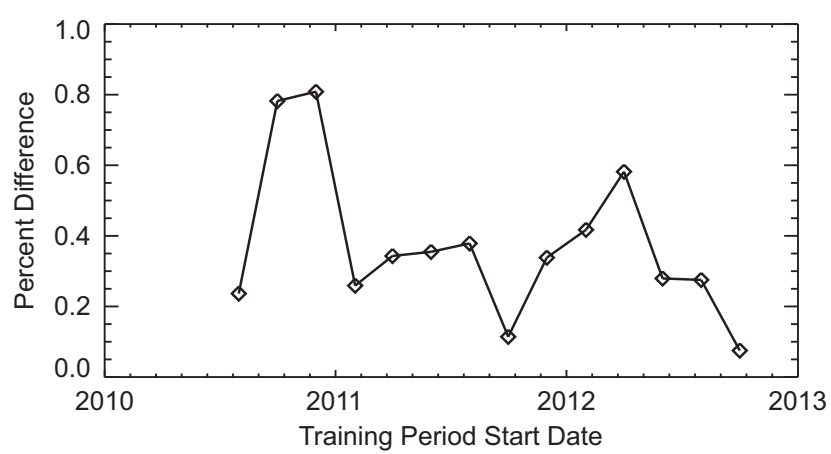

90-95 nm Band Percent Difference

(b)

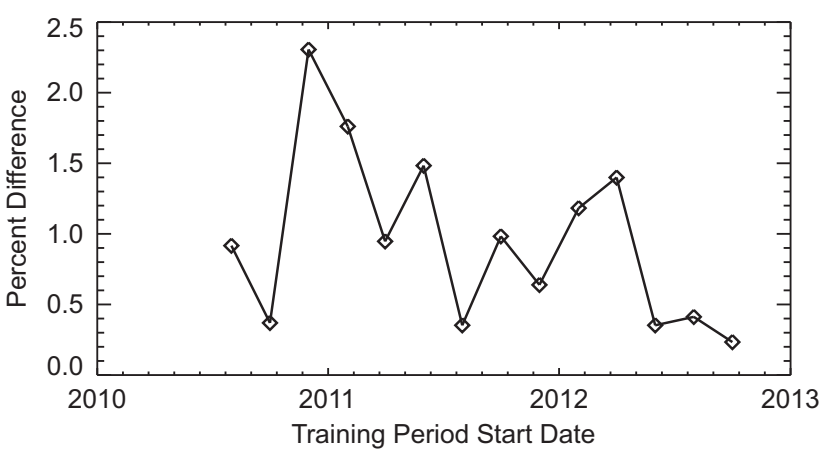

Fig. 5. When the overall irradiance level in the fitting interval more closely matches that of the two modeled weeks, the percent difference tends to be lower. Additionally, the percent difference is a minimum for the training period that includes the two predicted weeks and nearminimum for the training period just before the predicted weeks. Uncertainty in the reference data itself is a few percent. (a) Band with smallest percent difference, (b) band with largest percent difference.

Table 3. Start and end dates for the fitting intervals chosen to evaluate the effect of fitting interval choice on model accuracy.

\begin{tabular}{lcc}
\hline \hline Model number & Start date & End date \\
\hline 1 & August 1, 2010 & January 1, 2010 \\
2 & October 1, 2010 & March 1, 2011 \\
3 & December 1, 2010 & May 1, 2011 \\
4 & February 1, 2011 & July 1, 2011 \\
5 & April 1, 2011 & September 1, 2011 \\
6 & June 1, 2011 & November 1, 2011 \\
7 & August 1, 2011 & January 1, 2012 \\
8 & October 1, 2011 & March 1, 2012 \\
9 & December 1, 2011 & May 1, 2012 \\
10 & February 1, 2012 & July 1, 2012 \\
11 & April 1, 2012 & September 1, 2012 \\
12 & June 1, 2012 & November 1, 2012 \\
13 & August 1, 2012 & January 1, 2013 \\
14 & October 1, 2012 & March 1, 2013 \\
\hline
\end{tabular}

\subsection{Fixed training period evaluation}

One-year fixed fitting intervals demonstrated the effectiveness of SSPRING's linear combination proxy model method, but correlation between the model results and the observed data varied as the fitting interval was changed. In this section, we further explore the effect of the fitting interval choice on model effectiveness and test if the proximity of the fitting interval to the prediction date has an effect on the model-data correlation.

Creating 14 different overlapping fitting intervals of four months each allowed for sufficiently long fitting intervals. The dates to be predicted were the first 2 weeks of February 2013. The dates chosen as fitting intervals for each model are tabulated in Table 3. The first fitting interval is three full years before the test dates, and the last fitting interval includes the test dates.

After using the dates in Table 3 to create 14 sets of model parameters, we used those parameters to model the first two weeks of February 2013. Due to the small number of data points, the average percent difference between the model results and the data proved a more useful analytic than the correlation coefficient. In Figure 5 we plot the percent difference as a function of model number for several bands.

As expected, the fitting interval that includes the test dates produces the lowest percent difference. In general, the percent difference is also lower when the general irradiance level of the fitting interval more closely matches that of the test dates. We note that the difference between the first and last model's percent difference is greater for the 90-95 $\mathrm{nm}$ band, which generally has a much lower correlation than the $6-10 \mathrm{~nm}$ band. This indicates that the fitting interval choice could improve the poorly correlated bands more than the bands which already have high correlation, a promising way to improve the overall effectiveness of the model.

We also used the 14 sets of spectral coefficients to generate model results for the entire time period and then performed a reduced $\chi^{2}$ test on the results to determine if the error is Gaussian. We calculated the difference between the model results and the observed data, binned the differences into a histogram, fit a Gaussian to the histogram, and finally performed a reduced $\chi^{2}$ distribution test on the histogram and fitted Gaussian. If the model accurately represents the data, the error should be Gaussian and the reduced $\chi^{2}$ value should remain near unity.

Figure 6 shows the reduced $\chi^{2}$ values for particular bands plotted as a function of fitting interval date choice. We can pick minima and maxima from this graph to determine the optimal fitting interval choice.

In Figure 6, we see that both pictured bands have the same general shape, despite differences in specific fitting intervals. We note that these $\chi^{2}$ values are higher than desirable for many of the fitting intervals; this is likely because the model was trained on only a few months of reference data. Final model results use more than a four-month period to fit and do not suffer from this limitation.

Both bands have a minimum $\chi^{2}$ in 2011 . This is consistent with the fact that the 2011 year-long fitting interval is more effective than the 2012 year-long fitting interval for many wavelengths, and implies that the best choice of fixed fitting interval uses 2011 data. Much of this 2011 reference data is included in the variable fitting interval described in the next section.

\subsection{Variable training period results}

With only $\sim 3.5$ years of data, it is difficult to weigh the relative importance of a long fitting interval and a fitting interval using recent data. In this section, we approximate a long fitting 


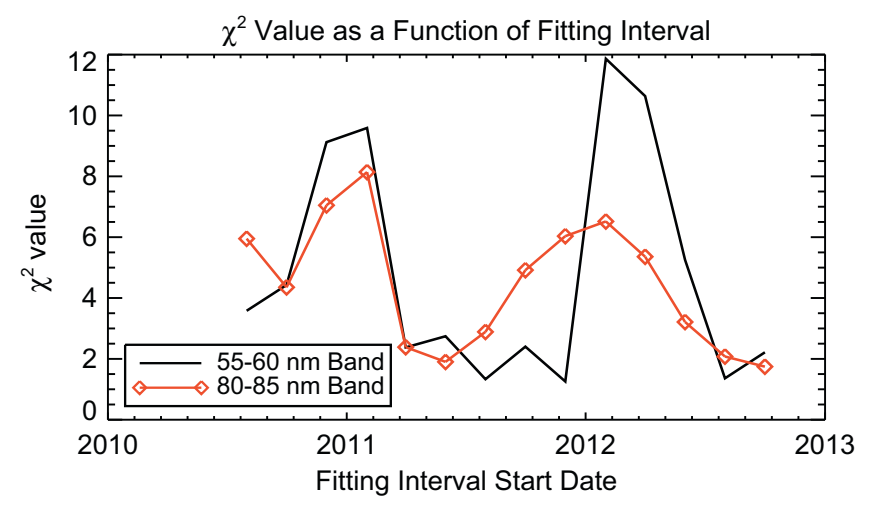

Fig. 6. Reduced $\chi^{2}$ as a function of fitting interval start date for two different bands. While the exact value differs for the two, the general shape of the curve is the same for these bands as well as the majority of the other 5-nm bands. For both, the minimum reduced $\chi^{2}$ occurs during 2011, showing that models including 2011 solar data tend to perform better than 2010- or 2012-based models.

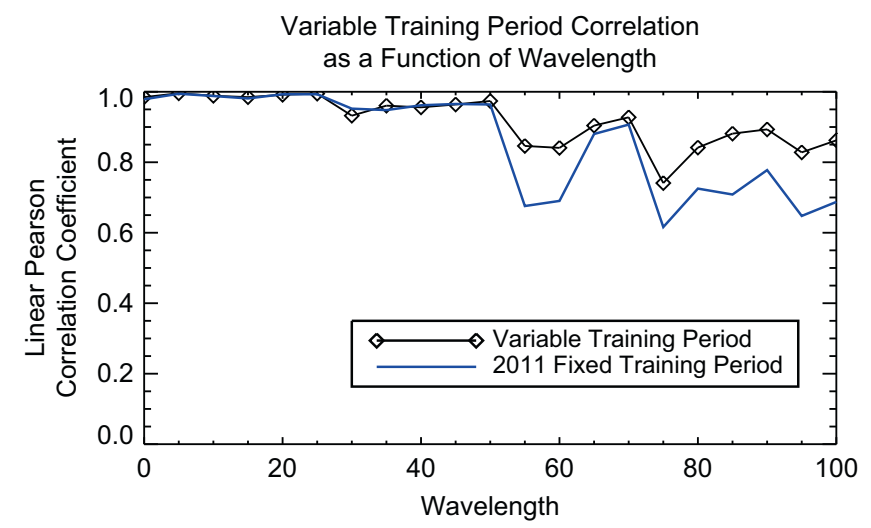

Fig. 7. Correlation coefficients for the variable fitting method and for the 2011 fixed fitting interval method, which had the higher correlation coefficients out of the 2 year-long fitting intervals tested. At longer wavelengths, it is clear that the variable fitting interval outperforms the fixed fitting intervals.

interval by using a variable training period that uses all days before the day to be predicted as a fitting interval.

To ensure that the variable model always has enough fitting data, we use all available 2010 data to fit the model for days in 2010. After December 31, 2010, we fit with all of the data available up until that day. For example, to model the EUV irradiance on April 3, 2012, the fitting interval spans from

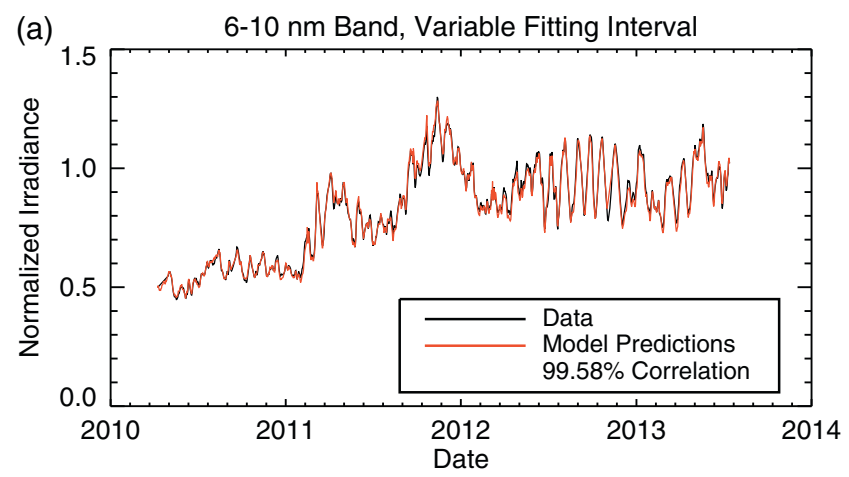

April 9, 2010, the first day we have GOES data, to April 2, 2012, the day before the day to be modeled. The fitting interval remains one day behind the prediction date, so the model includes information about the most recent solar activity but does not include future data. Due to the flexibility of the fitting interval selection with this model, we expect the model spectra produced to be day-to-day more accurate than using a fixed fitting interval.

As before, Figure 7 shows the linear Pearson correlation between the SSPRING results and the binned data. We also provide time series for the bands with highest and lowest correlation in Figure 8. In Figure 7 we see that for the $0.1-25 \mathrm{~nm}$ range, there is very little difference between the variable fitting interval and the fixed fitting interval correlations. However, the correlations split off longwards of $25 \mathrm{~nm}$ and it becomes clear that the variable fitting interval produces better results at long wavelengths. The difference is greatest in the 75-80 nm band, where the variable fitting interval has $17.84 \%$ better correlation than the 2012 one-year fitting interval.

Compared with Figure 3, the time series in Figure 8 demonstrate improved correlations for both the best and the worst wavelength band. The best band improved a slight amount, fairly undetectable in the plot. However, the lowest correlation band improved by almost $20 \%$, which leads to a noticeable increase in the quality of the modeled spectra. Along with the correlation coefficients in Figure 7, these time series help affirm that the variable fitting interval is the most accurate fitting interval tested.

Due to increases in accuracy, especially in the longer portion of the EUV spectrum, the variable fitting interval method is the best of the tested fitting intervals; this supports the idea that the best fitting interval uses a large amount of data, including recent data. The final version of SSPRING will use all available data. It is important to note, however, that additional SOLSTICE Mg II data is unavailable beyond the range used in the variable training model. The coefficients are therefore static beyond mid-2013, and model correlations will likely decrease as the prediction period moves farther away from the training period.

Table 4 lists model coefficients created with the entire fitting interval. Table 5 contains the mean and standard deviation of the mean for each coefficient over the days in the variable training period. The standard deviation of the mean is at least one order of magnitude smaller than the mean for all parameters; this indicates that the values are fairly stable in time over the 3.5 year fitting interval and suggests model predictions will not drift rapidly as the time between the fitting interval and prediction date increases.

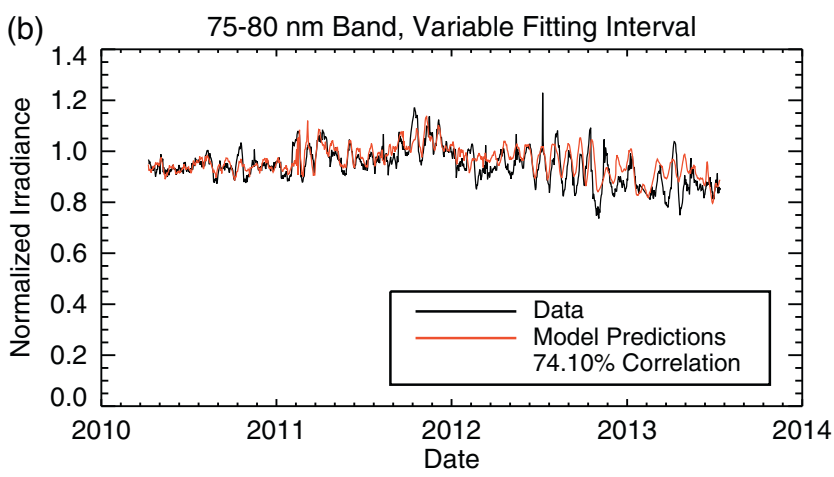

Fig. 8. Time series for the variable fitting interval model. Reference data uncertainties are a few percent, on the order of the size of the line used to plot. (a) Highest correlation band, (b) lowest correlation band. 
Table 4. Final model parameters for each wavelength band. Values are not normalized - when multiplied by the value of each proxy and added, an irradiance is produced.

\begin{tabular}{|c|c|c|c|c|c|c|c|}
\hline $\begin{array}{l}\text { Wavelength } \\
(\mathrm{nm})\end{array}$ & Offset & EUVS-B & EUVS-E & $\mathrm{Mg}$ II & Mg II average & $\begin{array}{l}\text { EUVS-E } \\
\text { average }\end{array}$ & $\begin{array}{c}\text { EUVS-B } \\
\text { average }\end{array}$ \\
\hline $0-6$ & $-6.42617 \mathrm{e}-05$ & $7.06405 \mathrm{e}-03$ & $-4.62286 \mathrm{e}-03$ & $9.49173 \mathrm{e}-04$ & $1.47148 \mathrm{e}-03$ & $5.12523 \mathrm{e}-03$ & $-3.55315 \mathrm{e}-03$ \\
\hline $6-10$ & $-4.04546 \mathrm{e}-05$ & $4.73700 \mathrm{e}-03$ & $-2.70627 \mathrm{e}-03$ & $1.44973 \mathrm{e}-04$ & $2.94192 \mathrm{e}-03$ & $2.00139 \mathrm{e}-03$ & $-1.95418 \mathrm{e}-03$ \\
\hline $10-15$ & $6.04379 \mathrm{e}-06$ & $4.45521 \mathrm{e}-03$ & $-2.24596 \mathrm{e}-03$ & $-6.02291 \mathrm{e}-04$ & $8.42154 \mathrm{e}-04$ & $1.01472 \mathrm{e}-03$ & $-7.97882 \mathrm{e}-04$ \\
\hline $15-20$ & $-3.61249 \mathrm{e}-06$ & $3.93912 \mathrm{e}-03$ & $-1.70728 \mathrm{e}-03$ & $-5.72592 \mathrm{e}-04$ & $1.66840 \mathrm{e}-03$ & $4.40539 \mathrm{e}-04$ & $-6.03789 \mathrm{e}-04$ \\
\hline $20-25$ & $-5.86938 \mathrm{e}-05$ & $4.29517 \mathrm{e}-03$ & $-2.03349 \mathrm{e}-03$ & $5.39989 \mathrm{e}-04$ & $2.99411 \mathrm{e}-03$ & $2.05809 \mathrm{e}-03$ & $-1.67364 \mathrm{e}-03$ \\
\hline $25-30$ & $-9.47743 \mathrm{e}-05$ & $4.69043 \mathrm{e}-03$ & $-2.47735 \mathrm{e}-03$ & $1.12381 \mathrm{e}-03$ & $6.12388 \mathrm{e}-03$ & $1.67788 \mathrm{e}-03$ & $-3.02795 \mathrm{e}-03$ \\
\hline $30-35$ & $-1.73432 \mathrm{e}-04$ & $2.91106 \mathrm{e}-03$ & $-9.46987 \mathrm{e}-04$ & $9.33763 \mathrm{e}-04$ & $1.75218 \mathrm{e}-02$ & $-8.94203 e-04$ & $-7.23309 \mathrm{e}-03$ \\
\hline $35-40$ & $-2.16379 \mathrm{e}-04$ & $3.83495 \mathrm{e}-03$ & $-2.05611 \mathrm{e}-03$ & $4.47723 e-04$ & $2.22088 \mathrm{e}-02$ & $-1.39458 \mathrm{e}-03$ & $-8.34631 \mathrm{e}-03$ \\
\hline $40-45$ & $-7.26451 \mathrm{e}-06$ & $4.53084 \mathrm{e}-03$ & $-3.03046 \mathrm{e}-03$ & $1.65238 \mathrm{e}-04$ & $1.36091 \mathrm{e}-03$ & $2.71422 \mathrm{e}-03$ & $-2.53264 \mathrm{e}-03$ \\
\hline $45-50$ & $3.17493 \mathrm{e}-05$ & $2.69783 \mathrm{e}-03$ & $-1.63611 \mathrm{e}-03$ & $8.45171 \mathrm{e}-04$ & $-3.72704 \mathrm{e}-03$ & $3.03315 \mathrm{e}-03$ & $3.94965 \mathrm{e}-05$ \\
\hline $50-55$ & $3.76448 \mathrm{e}-06$ & $3.06772 \mathrm{e}-03$ & $-1.86276 \mathrm{e}-03$ & $7.86446 \mathrm{e}-04$ & $-2.41385 \mathrm{e}-03$ & $3.72785 \mathrm{e}-03$ & $-5.27702 \mathrm{e}-04$ \\
\hline $55-60$ & $1.59101 \mathrm{e}-04$ & $1.45980 \mathrm{e}-03$ & $-5.54029 \mathrm{e}-04$ & $3.22083 \mathrm{e}-04$ & $-1.44124 \mathrm{e}-02$ & $2.20320 \mathrm{e}-03$ & $5.34420 \mathrm{e}-03$ \\
\hline $60-65$ & $2.13952 \mathrm{e}-04$ & $1.53776 \mathrm{e}-03$ & $-5.60600 \mathrm{e}-04$ & $1.52137 \mathrm{e}-04$ & $-1.64964 \mathrm{e}-02$ & $-2.10336 \mathrm{e}-04$ & $7.09364 \mathrm{e}-03$ \\
\hline $65-70$ & $1.21985 \mathrm{e}-04$ & $2.06389 \mathrm{e}-03$ & $-1.10970 \mathrm{e}-03$ & $3.34298 \mathrm{e}-04$ & $-8.67965 \mathrm{e}-03$ & $7.03226 \mathrm{e}-04$ & $3.12351 \mathrm{e}-03$ \\
\hline $70-75$ & $1.21347 \mathrm{e}-04$ & $1.82876 \mathrm{e}-03$ & $-9.31511 \mathrm{e}-04$ & $6.98381 \mathrm{e}-04$ & $-9.64268 \mathrm{e}-03$ & $8.51880 \mathrm{e}-04$ & $3.70568 \mathrm{e}-03$ \\
\hline $75-80$ & $1.96187 \mathrm{e}-04$ & $1.02472 \mathrm{e}-03$ & $-3.22738 \mathrm{e}-04$ & $4.90843 e-04$ & $-1.50470 \mathrm{e}-02$ & $4.79192 \mathrm{e}-04$ & $5.83441 \mathrm{e}-03$ \\
\hline $80-85$ & $1.71012 \mathrm{e}-04$ & $1.02671 \mathrm{e}-03$ & $-2.83592 \mathrm{e}-04$ & $9.52645 \mathrm{e}-04$ & $-1.47378 \mathrm{e}-02$ & $1.35059 \mathrm{e}-03$ & $5.59785 \mathrm{e}-03$ \\
\hline $85-90$ & $1.74547 \mathrm{e}-04$ & $1.01204 \mathrm{e}-03$ & $-2.46349 \mathrm{e}-04$ & $9.93918 \mathrm{e}-04$ & $-1.52968 \mathrm{e}-02$ & $7.94135 \mathrm{e}-04$ & $6.38254 \mathrm{e}-03$ \\
\hline $90-95$ & $1.66216 \mathrm{e}-04$ & $1.09385 \mathrm{e}-03$ & $-3.31018 \mathrm{e}-04$ & $9.49404 \mathrm{e}-04$ & $-1.41339 \mathrm{e}-02$ & $5.30995 \mathrm{e}-04$ & $5.98725 \mathrm{e}-03$ \\
\hline $95-100$ & $2.29911 \mathrm{e}-04$ & $1.04802 \mathrm{e}-03$ & $-1.99990 \mathrm{e}-04$ & $7.17327 \mathrm{e}-04$ & $-1.86072 \mathrm{e}-02$ & $1.23770 \mathrm{e}-04$ & $7.74255 \mathrm{e}-03$ \\
\hline $100-105$ & $1.90492 \mathrm{e}-04$ & $1.14364 \mathrm{e}-03$ & $-3.24574 \mathrm{e}-04$ & $8.44648 \mathrm{e}-04$ & $-1.58591 \mathrm{e}-02$ & $1.76448 \mathrm{e}-04$ & $6.87461 \mathrm{e}-03$ \\
\hline
\end{tabular}

\section{Comparison to FISM}

With the final fit parameters chosen (Table 4), we compare SSPRING to the results of the Flare Irradiance Spectral Model (FISM; Chamberlin et al. 2007). The daily version of FISM adds a solar cycle component and a solar rotation component to a minimum baseline irradiance value for each bin. The components added to each bin are determined by one optimal proxy (F10.7, Mg II index, or the flux in $0-4 \mathrm{~nm}, 36.5 \mathrm{~nm}$, $30.5 \mathrm{~nm}$, or Ly- $\alpha$ ) that is formed in the same layer of the solar atmosphere as the modeled wavelength band. If the optimal proxy is not available, FISM uses one of the other available proxies as a backup. The FISM model output is available on the LASP website at http://lasp.colorado.edu/lisird/fism/. For this comparison, we use the daily FISM version and rebin the 1-nm data into 5-nm bins to match the resolution of SSPRING.

There are several large differences in the construction of FISM and SSPRING. FISM uses only one proxy plus its centered 108-day mean value to model each band, while SSPRING uses five daily-value proxies and three trailing average proxies. SSPRING models the EUV irradiance in each band as a linear combination of the proxies, while FISM uses two equations to model the solar cycle and solar rotation variations, then adds those to a baseline value. FISM is currently produced in 1-nm bins and has versions with daily and 1-minute cadences, whereas SSPRING is currently produced with 5-nm bins and a daily time cadence. More work is required to increase SSPRING's time cadence to the $1 \mathrm{~min}$ achieved by FISM. This work will be non-trivial due to varying flare behavior.

Figure 9 shows the correlations for the two models to the reference data. Over the total 3.5 year test period, SSPRING has higher correlation with the data for most bands. There are five 5-nm bins where the FISM correlation is equal to or higher than SSPRING; the largest difference is $13 \%$. In the other fifteen 5-nm bins, SSPRING has better correlation than the FISM model; the largest difference is $41 \%$. The mean correlation coefficient across all wavelength bins is $92 \%$ for SSPRING, and $82 \%$ for FISM. Overall, this seems to indicate that for the time cadence and dates used by our model, SSPRING slightly outperforms FISM and may be a better indicator of daily EUV irradiances. However, these differences may be partially due to the two models using different training data sets below $40 \mathrm{~nm}$.

We also compared how well the two models perform during high and low solar activity periods. In practice, it is much more important to have an accurate model during times of high solar activity when large changes can be unexpected and could cause negative effects for a number of industries and consumers. Instead of looking at the correlation as a function of wavelength, we look at the average percent difference in all of the wavelength bands as a function of time. We studied one high-activity period, July to October 2012, and one low activity period, October 2010 to January 2011. We calculated the average percent difference between the model and the data across all wavelength bands for each day in the slowly- or quicklyvarying time period. Figure 10 shows the results.

During the low activity time period, the difference between the two models is much more pronounced than it is in the highactivity period. In the low activity period, SSPRING has a lower percent difference from the observations for all dates at an average of $10 \%$. During the high-activity time period, the results are more chaotic, with neither model clearly having a lower percent difference. Averaged across the time period, SSPRING has $2 \%$ lower percent difference than FISM. This difference is not particularly significant.

From this analysis, we see that although overall SSPRING performs better than FISM, much of this difference is due to better performance during periods of low solar activity. When it comes to the high-activity times that are more important to predict, the two models perform approximately equally. Furthermore, FISM provides higher temporal and spectral resolution than SSPRING offers at this time. It is also important to 
Table 5. Mean and standard deviation of the mean for all model parameters over the 3.5 year fitting interval. Values are not normalized - when multiplied by the value of each proxy and added, an irradiance is produced.

\begin{tabular}{|c|c|c|c|c|c|c|c|}
\hline $\begin{array}{l}\text { Wavelength } \\
(\mathrm{nm})\end{array}$ & Offset & EUVS-B & EUVS-E & Mg II & $\begin{array}{c}\text { Mg II } \\
\text { average }\end{array}$ & $\begin{array}{l}\text { EUVS-E } \\
\text { average }\end{array}$ & $\begin{array}{c}\text { EUVS-B } \\
\text { average }\end{array}$ \\
\hline \multirow[t]{2}{*}{$0-6$} & $-1.19314 \mathrm{e}-04$ & $5.58708 \mathrm{e}-03$ & $-3.82026 \mathrm{e}-03$ & $1.81731 \mathrm{e}-03$ & $7.57265 \mathrm{e}-03$ & $3.15330 \mathrm{e}-03$ & $-5.24481 \mathrm{e}-03$ \\
\hline & $\pm 1.40859 \mathrm{e}-06$ & $\pm 6.15760 \mathrm{e}-05$ & $\pm 4.01615 \mathrm{e}-05$ & $\pm 3.95360 \mathrm{e}-05$ & $\pm 1.02778 \mathrm{e}-04$ & $\pm 8.82349 \mathrm{e}-05$ & $\pm 5.40225 \mathrm{e}-05$ \\
\hline \multirow[t]{2}{*}{$6-10$} & $-2.63311 \mathrm{e}-05$ & $4.16340 \mathrm{e}-03$ & $-2.32838 \mathrm{e}-03$ & $1.86004 \mathrm{e}-04$ & $1.43278 \mathrm{e}-03$ & $1.62889 \mathrm{e}-03$ & $-8.28595 \mathrm{e}-04$ \\
\hline & $\pm 3.19436 \mathrm{e}-07$ & $\pm 3.36519 \mathrm{e}-05$ & $\pm 1.90653 \mathrm{e}-05$ & $\pm 4.33024 \mathrm{e}-06$ & $\pm 4.35070 \mathrm{e}-05$ & $\pm 4.00883 \mathrm{e}-05$ & $\pm 1.61451 \mathrm{e}-05$ \\
\hline \multirow[t]{2}{*}{$10-15$} & $9.67395 \mathrm{e}-06$ & $4.15551 \mathrm{e}-03$ & $-2.08318 \mathrm{e}-03$ & $-3.29636 \mathrm{e}-04$ & $-9.92936 \mathrm{e}-05$ & $8.06624 \mathrm{e}-04$ & $-9.65682 \mathrm{e}-05$ \\
\hline & $\pm 3.34506 \mathrm{e}-07$ & $\pm 3.32563 \mathrm{e}-05$ & $\pm 1.67141 \mathrm{e}-05$ & $\pm 5.81301 \mathrm{e}-06$ & $\pm 3.76983 \mathrm{e}-05$ & $\pm 4.74565 \mathrm{e}-05$ & $\pm 1.08528 \mathrm{e}-05$ \\
\hline \multirow[t]{2}{*}{$15-20$} & $1.19771 \mathrm{e}-05$ & $3.91888 \mathrm{e}-03$ & $-1.71030 \mathrm{e}-03$ & $-4.57236 \mathrm{e}-04$ & $-5.31489 \mathrm{e}-04$ & $5.49334 \mathrm{e}-04$ & $4.81356 \mathrm{e}-04$ \\
\hline & $\pm 6.42133 \mathrm{e}-07$ & $\pm 3.31921 \mathrm{e}-05$ & $\pm 1.52704 \mathrm{e}-05$ & $\pm 4.44501 \mathrm{e}-06$ & $\pm 3.00635 \mathrm{e}-05$ & $\pm 6.92161 \mathrm{e}-05$ & $\pm 2.13226 \mathrm{e}-05$ \\
\hline \multirow[t]{2}{*}{$20-25$} & $-3.85482 \mathrm{e}-05$ & $4.19705 \mathrm{e}-03$ & $-2.00912 \mathrm{e}-03$ & $6.70752 \mathrm{e}-05$ & $2.07654 \mathrm{e}-03$ & $1.30788 \mathrm{e}-03$ & $-7.06145 \mathrm{e}-04$ \\
\hline & $\pm 4.26173 \mathrm{e}-07$ & $\pm 3.44334 \mathrm{e}-05$ & $\pm 1.66706 \mathrm{e}-05$ & $\pm 8.40989 \mathrm{e}-06$ & $\pm 4.19641 \mathrm{e}-05$ & $\pm 4.51724 \mathrm{e}-05$ & $\pm 1.40254 \mathrm{e}-05$ \\
\hline \multirow[t]{2}{*}{$25-30$} & $-4.99135 \mathrm{e}-05$ & $4.44488 \mathrm{e}-03$ & $-2.28564 \mathrm{e}-03$ & $4.14720 \mathrm{e}-04$ & $2.83153 \mathrm{e}-03$ & $1.29582 \mathrm{e}-03$ & $-1.21571 \mathrm{e}-03$ \\
\hline & $\pm 8.26882 \mathrm{e}-07$ & $\pm 3.57821 \mathrm{e}-05$ & $\pm 1.83867 \mathrm{e}-05$ & $\pm 1.13666 \mathrm{e}-05$ & $\pm 7.15158 \mathrm{e}-05$ & $\pm 2.56447 \mathrm{e}-05$ & $\pm 3.15318 \mathrm{e}-05$ \\
\hline \multirow[t]{2}{*}{$30-35$} & $-3.54392 \mathrm{e}-05$ & $3.51785 \mathrm{e}-03$ & $-1.11613 \mathrm{e}-03$ & $-8.42005 \mathrm{e}-04$ & $4.75016 \mathrm{e}-03$ & $5.39014 \mathrm{e}-04$ & $-2.16895 \mathrm{e}-03$ \\
\hline & $\pm 1.96011 \mathrm{e}-06$ & $\pm 3.71876 \mathrm{e}-05$ & $\pm 1.60196 \mathrm{e}-05$ & $\pm 3.18992 \mathrm{e}-05$ & $\pm 1.34078 \mathrm{e}-04$ & $\pm 4.90561 \mathrm{e}-05$ & $\pm 7.04941 \mathrm{e}-05$ \\
\hline \multirow[t]{2}{*}{$35-40$} & $-6.88350 \mathrm{e}-05$ & $4.07662 \mathrm{e}-03$ & $-1.94210 \mathrm{e}-03$ & $-6.20909 \mathrm{e}-04$ & $8.51927 \mathrm{e}-03$ & $-1.24676 \mathrm{e}-03$ & $-2.13196 \mathrm{e}-03$ \\
\hline & $\pm 2.50331 \mathrm{e}-06$ & $\pm 3.48722 \mathrm{e}-05$ & $\pm 1.60244 \mathrm{e}-05$ & $\pm 1.69936 \mathrm{e}-05$ & $\pm 2.45032 \mathrm{e}-04$ & $\pm 5.11822 \mathrm{e}-05$ & $\pm 1.13757 \mathrm{e}-04$ \\
\hline \multirow[t]{2}{*}{$40-45$} & $-4.81382 \mathrm{e}-05$ & $2.99928 \mathrm{e}-03$ & $-2.15061 \mathrm{e}-03$ & $1.77964 \mathrm{e}-03$ & $5.74169 \mathrm{e}-03$ & $-2.01440 \mathrm{e}-04$ & $-3.02636 \mathrm{e}-03$ \\
\hline & $\pm 1.34496 \mathrm{e}-06$ & $\pm 3.18922 \mathrm{e}-05$ & $\pm 2.03318 \mathrm{e}-05$ & $\pm 3.42264 \mathrm{e}-05$ & $\pm 1.20222 \mathrm{e}-04$ & $\pm 4.49862 \mathrm{e}-05$ & $\pm 5.23460 \mathrm{e}-05$ \\
\hline \multirow[t]{2}{*}{$45-50$} & $-1.94667 \mathrm{e}-05$ & $1.79897 \mathrm{e}-03$ & $-1.19909 \mathrm{e}-03$ & $1.90169 \mathrm{e}-03$ & $1.51067 \mathrm{e}-03$ & $1.21737 \mathrm{e}-03$ & $-1.51840 \mathrm{e}-03$ \\
\hline & $\pm 9.85663 \mathrm{e}-07$ & $\pm 1.94370 \mathrm{e}-05$ & $\pm 1.13825 \mathrm{e}-05$ & $\pm 2.32360 \mathrm{e}-05$ & $\pm 1.03170 \mathrm{e}-04$ & $\pm 4.11927 \mathrm{e}-05$ & $\pm 3.90093 \mathrm{e}-05$ \\
\hline \multirow[t]{2}{*}{$50-55$} & $-3.35590 \mathrm{e}-05$ & $2.12914 \mathrm{e}-03$ & $-1.36839 \mathrm{e}-03$ & $1.76202 \mathrm{e}-03$ & $1.58908 \mathrm{e}-03$ & $1.76462 \mathrm{e}-03$ & $-1.33589 \mathrm{e}-03$ \\
\hline & $\pm 7.82210 \mathrm{e}-07$ & \pm 2.1 & $\pm 1.27663 \mathrm{e}-05$ & $\pm 2.25724 \mathrm{e}-05$ & $\pm 7.40677 \mathrm{e}-05$ & $\pm 3.81889 \mathrm{e}-05$ & $228 \mathrm{e}-05$ \\
\hline \multirow[t]{2}{*}{$55-60$} & $4.05600 \mathrm{e}-05$ & $1.18207 \mathrm{e}-03$ & $-5.98232 \mathrm{e}-04$ & $1.71613 e-03$ & $-6.38722 \mathrm{e}-03$ & $3.73891 \mathrm{e}-03$ & $9.78695 \mathrm{e}-04$ \\
\hline & $\pm 1.93593 \mathrm{e}-06$ & $\pm 1.06715 \mathrm{e}-05$ & $\pm 6.04107 \mathrm{e}-06$ & $\pm 2.93829 \mathrm{e}-05$ & $\pm 1.26416 \mathrm{e}-04$ & $\pm 6.80817 \mathrm{e}-05$ & $\pm 7.25111 \mathrm{e}-05$ \\
\hline \multirow[t]{2}{*}{$60-65$} & $5.84035 \mathrm{e}-05$ & $1.13361 \mathrm{e}-03$ & $-6.09801 \mathrm{e}-04$ & $1.82744 \mathrm{e}-03$ & $-4.94278 \mathrm{e}-03$ & $9.30378 \mathrm{e}-04$ & $1.43943 \mathrm{e}-03$ \\
\hline & $\pm 2.65779 \mathrm{e}-06$ & $\pm 1.10618 \mathrm{e}-05$ & $\pm 5.75132 \mathrm{e}-06$ & $\pm 3.46112 \mathrm{e}-05$ & $\pm 1.67985 \mathrm{e}-04$ & $\pm 7.82188 \mathrm{e}-05$ & $\pm 9.59565 \mathrm{e}-05$ \\
\hline \multirow[t]{2}{*}{$65-70$} & $3.50155 \mathrm{e}-05$ & $1.39337 \mathrm{e}-03$ & $-8.48923 \mathrm{e}-04$ & $1.78318 \mathrm{e}-03$ & $-2.35701 \mathrm{e}-03$ & $6.75712 \mathrm{e}-04$ & $2.88143 \mathrm{e}-04$ \\
\hline & $\pm 1.60811 \mathrm{e}-06$ & $\pm 1.39225 \mathrm{e}-05$ & $\pm 7.32983 \mathrm{e}-06$ & $\pm 2.91884 \mathrm{e}-05$ & $\pm 1.11647 \mathrm{e}-04$ & $\pm 3.54816 \mathrm{e}-05$ & $\pm 5.64359 \mathrm{e}-05$ \\
\hline \multirow[t]{2}{*}{$70-75$} & $1.76742 \mathrm{e}-05$ & $1.23443 \mathrm{e}-03$ & $-7.52727 \mathrm{e}-04$ & $2.12585 \mathrm{e}-03$ & $-2.11733 e-03$ & $1.25490 \mathrm{e}-03$ & $1.94774 \mathrm{e}-04$ \\
\hline & $\pm 1.76558 \mathrm{e}-06$ & $\pm 1.27405 \mathrm{e}-05$ & $\pm 6.62558 \mathrm{e}-06$ & $\pm 3.01251 \mathrm{e}-05$ & $\pm 1.11725 \mathrm{e}-04$ & $\pm 4.92750 \mathrm{e}-05$ & $\pm 6.02420 \mathrm{e}-05$ \\
\hline \multirow[t]{2}{*}{$75-80$} & $5.46471 \mathrm{e}-05$ & $4.42361 \mathrm{e}-04$ & $-2.09970 \mathrm{e}-04$ & $2.25326 \mathrm{e}-03$ & $-5.08675 \mathrm{e}-03$ & $1.60369 \mathrm{e}-03$ & $9.70616 \mathrm{e}-04$ \\
\hline & $\pm 2.23856 \mathrm{e}-06$ & $\pm 1.14485 \mathrm{e}-05$ & $\pm 5.45397 \mathrm{e}-06$ & $\pm 3.72513 \mathrm{e}-05$ & $\pm 1.31260 \mathrm{e}-04$ & $\pm 6.93033 \mathrm{e}-05$ & $\pm 7.46772 \mathrm{e}-05$ \\
\hline \multirow[t]{2}{*}{$80-85$} & $2.91539 \mathrm{e}-05$ & $6.15804 \mathrm{e}-04$ & $-3.09063 \mathrm{e}-04$ & $2.45166 \mathrm{e}-03$ & $-3.86461 \mathrm{e}-03$ & $2.06427 \mathrm{e}-03$ & $4.39589 \mathrm{e}-04$ \\
\hline & $\pm 2.20246 \mathrm{e}-06$ & $\pm 7.78925 \mathrm{e}-06$ & $\pm 3.43226 \mathrm{e}-06$ & $\pm 3.37231 \mathrm{e}-05$ & $\pm 1.48411 \mathrm{e}-04$ & $\pm 6.01722 \mathrm{e}-05$ & $\pm 7.98243 \mathrm{e}-05$ \\
\hline \multirow[t]{2}{*}{$85-90$} & $2.90166 \mathrm{e}-05$ & $7.04584 \mathrm{e}-04$ & $-3.64855 \mathrm{e}-04$ & $2.35898 \mathrm{e}-03$ & $-3.41525 \mathrm{e}-03$ & $1.17958 \mathrm{e}-03$ & $8.34713 \mathrm{e}-04$ \\
\hline & $\pm 2.08065 \mathrm{e}-06$ & $\pm 7.10269 \mathrm{e}-06$ & $\pm 4.18138 \mathrm{e}-06$ & $\pm 3.00271 \mathrm{e}-05$ & $\pm 1.57785 \mathrm{e}-04$ & $\pm 4.15164 \mathrm{e}-05$ & $\pm 7.92331 \mathrm{e}-05$ \\
\hline \multirow[t]{2}{*}{$90-95$} & $3.22496 \mathrm{e}-05$ & $8.81250 \mathrm{e}-04$ & $-4.87212 \mathrm{e}-04$ & $2.10896 \mathrm{e}-03$ & $-3.16698 \mathrm{e}-03$ & $9.98522 \mathrm{e}-04$ & $7.83766 \mathrm{e}-04$ \\
\hline & $\pm 1.90424 \mathrm{e}-06$ & $\pm 8.00485 \mathrm{e}-06$ & $\pm 5.14244 \mathrm{e}-06$ & $\pm 2.58093 \mathrm{e}-05$ & $\pm 1.44256 \mathrm{e}-04$ & $\pm 3.88194 \mathrm{e}-05$ & $\pm 7.36882 \mathrm{e}-05$ \\
\hline \multirow[t]{2}{*}{ 95-100 } & $5.50652 \mathrm{e}-05$ & $7.95736 \mathrm{e}-04$ & $-4.29084 \mathrm{e}-04$ & $2.20643 \mathrm{e}-03$ & $-3.74107 e-03$ & $2.25695 \mathrm{e}-04$ & $9.71146 \mathrm{e}-04$ \\
\hline & $\pm 2.48525 \mathrm{e}-06$ & $\pm 8.52740 \mathrm{e}-06$ & $\pm 5.48696 \mathrm{e}-06$ & $\pm 3.09760 \mathrm{e}-05$ & $\pm 1.95972 \mathrm{e}-04$ & $\pm 4.25452 \mathrm{e}-05$ & $\pm 9.50481 \mathrm{e}-05$ \\
\hline \multirow[t]{2}{*}{$100-105$} & $3.80014 \mathrm{e}-05$ & $8.20747 \mathrm{e}-04$ & $-4.60687 \mathrm{e}-04$ & $2.18710 \mathrm{e}-03$ & $-2.79669 \mathrm{e}-03$ & $9.55720 \mathrm{e}-05$ & $1.01174 \mathrm{e}-03$ \\
\hline & $\pm 2.11495 \mathrm{e}-06$ & $\pm 9.52858 \mathrm{e}-06$ & $\pm 5.44085 \mathrm{e}-06$ & $\pm 2.92446 \mathrm{e}-05$ & $\pm 1.67610 \mathrm{e}-04$ & $\pm 3.51286 \mathrm{e}-05$ & $\pm 8.04822 \mathrm{e}-05$ \\
\hline
\end{tabular}

note that while SSPRING was trained over 2010-2013, FISM was trained on TIMED SEE data from 2002 to 2005 (P.C. Chamberlin, private communication). The long time interval between FISM's training period and the test dates likely contributed to the decreased accuracy of FISM. It is unclear if SSPRING would continue to outperform FISM during low solar activity if SSPRING's training period was as far away from the test days as FISM's.

\section{Model expansions}

Expanding SSPRING to other wavelength bands or somewhat longer wavelengths is feasible due to the model construction. However, if a split reference data set between EVE and SEE continues to be used for a finer wavelength resolution, differences between the spectral resolution of the two instruments

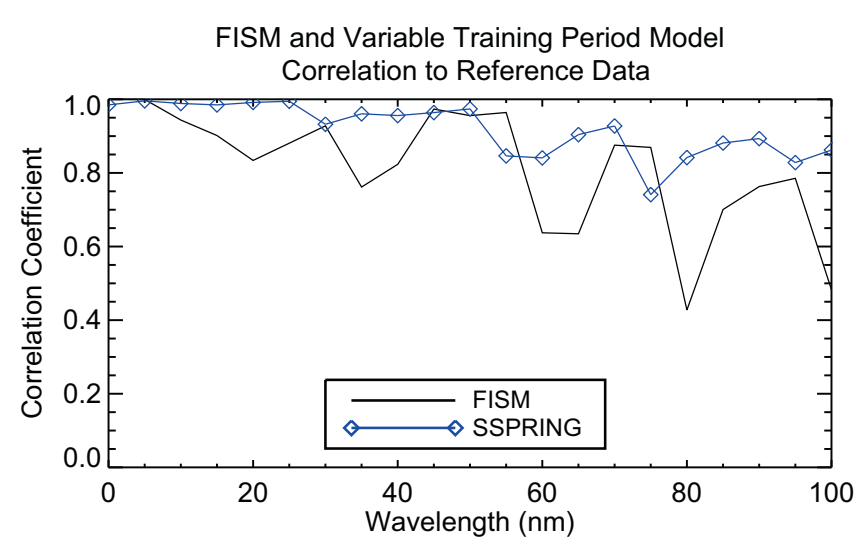

Fig. 9. Correlation coefficients for FISM and the variable fitting interval SSPRING model as a function of wavelength. 
(a)

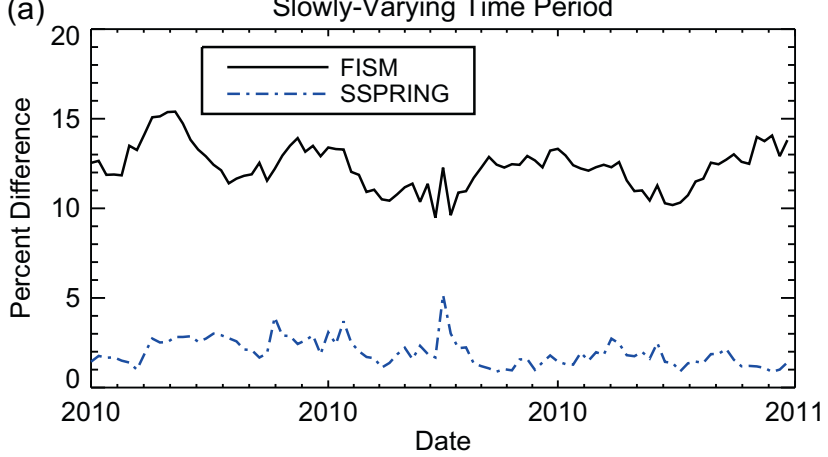

(b)

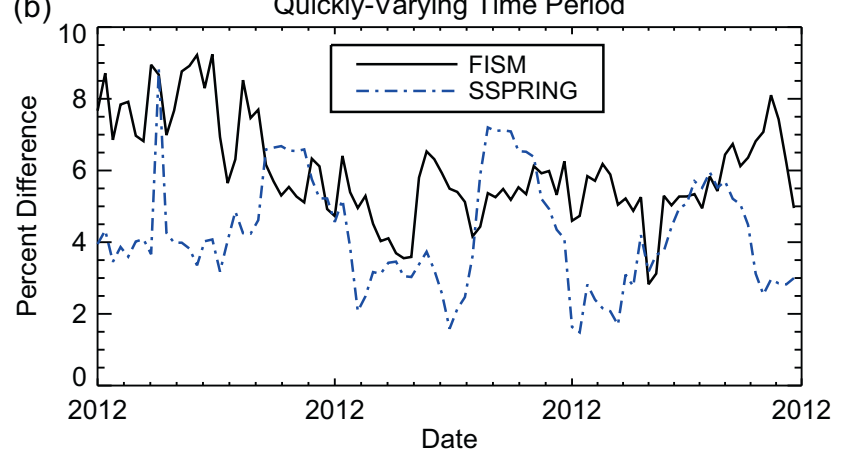

Fig. 10. Percent difference between model and measurements as a function of time during two different time periods. Reference data uncertainties are a few percent. (a) Low solar activity, (b) high solar activity.

begin to become important. We expect that this technique could be used to model at least to Ly- $\alpha$. If wavelengths much longer than this are used, more input data sets will likely be required to capture solar variability in the new wavelength regions.

It is also possible to use this concept to model shorter timescales, such as hourly or even minute-by-minute cadences. Again, with this increase in resolution we could no longer assume parallels between the EVE and SEE reference data sets. There may also be additional challenges to a shorter timescale model due to the varying spectral behavior of different types of flares.

The bandpasses on the new GOES-R EUV instrument will be constructed using a similar technique as SSPRING, although on much shorter timescales. Eparvier et al. (2009) describe the high spectral resolution measurements in three EUV bandpasses that will be measured by GOES-R, as well as the NOAA criteria of a 30-second time resolution and $20 \%$ accuracy. SSPRING can serve as a proof of concept for a linear combination proxy model in the EUV spectrum such as the one that will be used on GOES-R. For near term model expansions, it is unlikely that the test time period will be expanded beyond the early part of 2014 due to the degradation of EVE MEGS-B and the May 2014 power failure of MEGS-A.

\section{Conclusions}

In this paper, we present a new daily proxy model for EUV irradiance that spans the $0.1-105 \mathrm{~nm}$ range in 5-nm bins. The model is constructed using a Levenberg-Marquardt least squares fitting algorithm which determines scalar weighting coefficients that best produce observed daily EUV irradiance with a linear combination of input data series. The input data series used were two EUV channels and two XRS channels on the NOAA GOES satellites, the Mg II index from SORCE SOLSTICE, and 40-day trailing averages of the GOES EUV channels and $\mathrm{Mg}$ II index. We tested several different fitting intervals to determine which provided the best results. First, we validated the basic model concept using a one-year fixed fitting interval. Second, we "marched" the fitting interval forward in time, closer to the prediction dates, to determine that in general it is beneficial to include recent solar data in the calculation of the model. Finally, we tested a variable fitting interval model that fit to all of the data available until the modeled day. This variable fitting interval method provided the best results. The correlation between the model results and observed irradiance varied with the wavelength, but was above $90 \%$ for 13 of the 21 bands, and was above $80 \%$ for all but one band. We also compared this model to the Flare Irradiance Spectral Model and saw that, as a whole, SSPRING had better correlation with the data. This difference was mainly in the low solar activity time periods, however, and there was not a significant improvement in model results during high solar activity time periods. It is possible to expand SSPRING to have higher wavelength or temporal resolution, but further work needs to be done to model shorter timescales.

Acknowledgements. We would like to thank the referees and editors for their help in improving this manuscript. This work was supported by NASA Grant NNX13AI25A S03 (MUSSIC) and NSF Grant AGS-1157020 (REU), both at the University of Colorado Boulder. We also thank Tom Woods and the instrument teams at LASP for the TIMED/SEE, SDO/EVE, and SORCE/SOLSTICE data used in this investigation. The FISM data used in this study was retrieved from LISIRD, and we thank LASP for making this web service available to the community. The GOES EUV data is from http://ngdc.noaa.gov/stp/satellite/goes/dataaccess.html and is the work of many people at NOAA. The editor thanks two anonymous referees for their assistance in evaluating this paper.

\section{References}

Cessateur, G., T. Dudok de Wit, M. Kretzschmar, J. Lilensten, J.-F. Hochedez, and M. Snow. Monitoring the solar UV irradiance spectrum from the observations of a few passbands. Astron. Astrophys., 528, A68, 2011,

DOI: $10.1051 / 0004-5351 / 201015903$.

Chamberlin, P.C., T.N. Woods, and F.G. Eparvier. Flare Irradiance Spectral Model (FISM): daily component algorithms and results. Space Weather, 5, S07005, 2007, DOI: 10.1029/2007SW000316.

Dudok de Wit, T., S. Bruinsma, and K. Shibasaki. Synoptic radio observations as proxies for upper atmospheric modeling. J. Space Weather Space Clim., 4, A06, 2014, DOI: $10.1051 / \mathrm{swsc} / 2014003$.

Eparvier, F.G., D. Crotser, A.R. Jones, W.E. McClintock, M. Snow, and T.N. Woods. The Extreme Ultraviolet Sensor (EUVS) for GOES-R. SPIE Proceedings, 7438, 2009, DOI: $10.1117 / 12.826445$.

EVE News 19 May 2010, http://lasp.colorado.edu/home/eve/2010/ 05/19/operations-change-for-megs-b-and-megs-p/

EVE News 28 May 2010, http://asp.colorado.edu/home/eve/2014/ 05/28/eve-megs-a-power-anomaly/?doing_wp_cron=1454706787. 2851901054382324218750

Fontenla, J., E. Landi, M. Snow, and T. Woods. Far- and extremeUV solar spectral irradiance and radiation from simplified atmospheric physical models. Sol. Phys, 289, 515-544, 2014, DOI: $10.1007 / \mathrm{s} 11207-013-0431-4$.

Heath, D.F., and B.M. Schlesinger. The Mg $280 \mathrm{~nm}$ doublet as a monitor of changes in the solar ultraviolet irradiance. J. Geophys. Res., 91, 8672-8682, 1986, DOI: 10.1029/JD091iD08p08672. 
Hinteregger, H.E., and K. Fukui. Observational, reference and model data on solar EUV, from measurements on AE-E. J. Geophys. Res., 91, 1147-1150, 1981, DOI: $10.1029 / \mathrm{GL} 008 \mathrm{i} 011 \mathrm{p} 01147$.

Lean, J.L., H.P. Warren, J.T. Mariska, and J. Bishop. A new model of solar EUV irradiance variability: 2. Comparisons with empirical models and observations and implications for space weather. J. Geophys. Res., 108 (A2), 1059, 2003, DOI: $10.1029 / 2001$ JA009238.

Markwardt, C.B. Non-linear least squares fitting in IDL with MPFIT. In: D. Bohlender, P. Dowler, and D. Durand, Proc. Astronomical Data Analysis Software and Systems XVIII, Quebec, Canada, ASP Conference Series, Vol. 411, Astronomical Society of the Pacific: San Francisco, 251-254, 2009.

McClintock, W.E., M. Snow, and T.N. Woods. Solar-Stellar Irradiance Comparison Experiment II (SOLSTICE II): prelaunch and on-orbit calibrations. Sol. Phys., 230, 259-294, 2005, DOI: 10.1007/0-387-37625-9_13.

Richards, P.G., J.A. Fennelly, and D.G. Torr. EUVAC: a solar EUV flux model for aeronomic calculations J. Geophys. Res., 99, 8981-8992, 1994, DOI: 10.1029/94JA00518.

Richards, P.G., T.N. Woods, and W.K. Peterson. HEUVAC: a new high resolution solar EUV proxy model. Adv. Space Res., 37, 315-322, 2006, DOI: 10.1016/j.asr.2005.06.031.

Rottman, G. The SORCE mission. Sol. Phys., 230, 7-25, 2005, DOI: $10.1007 / 0-387-37625-92$.

Snow, M., W.E. McClintock, T.N. Woods, O.R. White, J.W. Harder, and G. Rottman. The Mg II index from SORCE. Sol. Phys., 230, 325-344, 2005, DOI: 10.1007/0-387-37625-9_15.

Solomon, S.C., and L. Qian. Solar extreme-ultraviolet irradiance for general circulation models. J. Geophys. Res., 110, A10306, 2005, DOI: $10.1029 / 2005 J A 011160$.

Tapping, K.F. The $10.7 \mathrm{~cm}$ solar radio flux $\left(F_{10.7}\right)$. Space Weather, 11, 394-406, 2013, DOI: 10.1002/swe.20064.

Tobiska, W.K., T.N. Woods, F.G. Eparvier, R. Viereck, L. Floyd, D. Bower, G.J. Rottman, and O.R. White. The SOLAR2000 empirical solar irradiance model and forecast tool. J. Atmos. Sol. Terr. Phys., 62, 1233-1250, 2000,

DOI: 10.1016/S1364-6826(00)00070-5.

Tobiska, W.K. SOLAR2000 irradiances for climate change research, aeronomy, and space system engineering. Adv. Space Res., 34, 1736-1746, 2004, DOI: 10.1016/j.asr.2003.06.032.

Viereck, R., L. Puga, D. McMullin, D. Judge, M. Weber, and W.K. Tobiska. The Mg II index: a proxy for solar EUV. Geophys. Res. Lett., 28 (7), 1343-1346, 2001, DOI: 10.1016/j.asr.2003.06.032.

Viereck, R., F. Hanser, J. Wise, S. Guha, A. Jones, D. McMullin, S. Plunket, D. Strickland, and S. Evans. Solar extreme ultraviolet irradiance observations from GOES: design characteristics and initial performance. Proc. SPIE, 6689, Solar Physics and Space Weather Instrumentation II, 66890K, 2007, DOI: $10.1117 / 12.734886$

Warren, H.P. A solar minimum irradiance spectrum for wavelengths below $1200 \AA$ A. Astrophys. J., 157, 147-173, 2005, DOI: $10.1086 / 427171$.

Warren, H.P., J.T. Mariska, and J. Lean. A new model of solar EUV irradiance variability 1. Model formulation. J. Geophys. Res., 106, 15745-15757, 2001, DOI: 10.1029/2000JA000282.

Woods, T.N., F.G. Eparvier, S.M. Bailey, P.C. Chamberlin, J. Lean, G.J. Rottman, S.C. Solomon, W.K. Tobiska, and D.L. Woodraska. Solar EUV Experiment (SEE): mission overview and first results. J. Geophys. Res., 110, A01312, 2005, DOI: $10.1029 / 2004 J A 010765$.

Woods, T.N., P.C. Chamberlin, J.W. Harder, R. Hock, M. Snow, F.G. Eparvier, J. Fontenla, W.E. McClintock, and E.C. Richard. Solar Irradiance Reference Spectra (SIRS) for the 2008 Whole Heliosphere Interval (WHI). Geophys. Res. Lett., 36, L01101, 2009, DOI: 10.1029/2008GL036373.

Woods, T.N., F.G. Eparvier, R. Hock, A.R. Jones, D. Woodraska, et al. Extreme ultraviolet variability experiment (EVE) on the solar dynamics observatory (SDO): overview of science objectives, instrument design, data products, and model developments. Sol. Phys., 275, 115-143, 2012, DOI: 10.1007/s11207-009-9487-6.

Cite this article as: Suess K, Snow M, Viereck R \& Machol J. Solar Spectral Proxy Irradiance from GOES (SSPRING): a model for solar EUV irradiance. J. Space Weather Space Clim., 6, A10, 2016, DOI: 10.1051/swsc/2016003. 